\author{
Ursula Wolfa \\ Peter Heusser ${ }^{b}$ \\ Thomas Cerny ${ }^{c}$ \\ Marc R. Schläppi ${ }^{c}$
}

Zwischen 14,8 und 73,1\% der Patienten und Patientinnen mit Krebserkrankungen wenden im Verlauf ihrer Erkrankung neben der konventionellen Medizin komplementärmedizinische Massnahmen an $[1,2]$. Motivationen dafür sind, dass die Patienten eine nicht nur auf die Erkrankung fokussierte Therapie wünschen, sondern auch eine Unterstützung ihrer Gesundungskräfte und eine Verbesserung der Lebensqualität. Integrative Onkologie bedeutet die synergistische Anwendung von konventionell-medizinischen und komplementärmedizinischen Massnahmen, im Gegensatz zu nur additiver Anwendung von Komplementärmedizin [3]. Gerade weil komplementärmedizinische Massnahmen von vielen Krebspatienten angewendet werden, besteht im Gebiet der Integrativen Onkologie grosser Forschungsbedarf, insbesondere in den Bereichen Wirksamkeit, Interaktionen, Sicherheit und auch Kosteneffektivität. Um diesem Forschungsbedarf besser entsprechen zu können, ist auch eine Erweiterung und Weiterentwickelung der Kommunikation zwischen den Vertretern der verschiedenen Fachrichtungen in der Onkologie notwendig.

Ziele des Symposiums waren daher einerseits die Identifikation wichtiger, klinisch relevanter Forschungsfragen, die Formulierung der notwendigen

aUniversität Bern, Schweiz

bUniversität Witten-Herdecke, Deutschland

'Kantonsspital St. Gallen, Schweiz

\title{
Erstes Symposium für Integrative Onkologie und Forschung in der Schweiz
}

Vom 30.-31. Oktober 2009 fand das erste Symposium «Integrative Onkologie und Forschung - Neue Aspekte und Netzwerkbildung» am Kantonsspital in St. Gallen statt. Das Symposium wurde in Kooperation vom Kantonsspital St. Gallen (Prof. Thomas Cerny, Dr. Marc Schläppi), der Universität WittenHerdecke (Dr. Peter Heusser) und der Kollegialen Instanz für Komplementärmedizin der Universität Bern (Dr. Ursula Wolf) organisiert und durchgeführt.

Schritte zur Ermöglichung einer qualitativ hochstehenden Forschung im Bereich der Integrativen Onkologie sowie die Bildung eines interdisziplinären Forschungsnetzwerkes für Integrative Onkologie, in dem sich die Vertreter der verschiedenen Fachrichtungen zu einem Austausch und zur Förderung gemeinsamer Forschungsprojekte begegnen können. Die Schwerpunkte des Symposiums waren: 1. Methodologie; 2. Phytotherapie und Spurenelemente; 3. Misteltherapie; 4. Akupunktur; 5. Mind/Body, Achtsamkeit; 6. Kunsttherapien und 7. Pflegeanwendungen und Rolle der Pflege. Teilnehmende waren ÄrztInnen, TherapeutInnen, Pflegende und Forschende.

In jedes Thema wurde zunächst im Plenum mit einem Impulsreferat eingeführt. Im Anschluss wurden die jeweiligen Themengebiete unter Leitung der Referentinnen und Referenten in Workshops mit dem Ziel, möglichst konkrete Forschungsfragen entwickeln zu können, vertieft und in einem abschliessenden Plenum vorgestellt. Für die Impulsreferate und Leitung der Workshops konnten namhafte Referentinnen und Referenten gewonnen werden: Prof. Claudia Witt, Berlin; Dr. Brigitte Ausfeld-Hafter,
Bern; Dietrich von Bonin, Bern; Dr. Roman Huber, Freiburg i.Br.; Dr. Jutta Hübner, Kassel; Prof. Reinhard Saller, Zürich; Dr. Astrid Baege, Zürich; Prof. Harald Walach, Northampton; Prof. Harald Gruber, Alfter bei Bonn; Dr. Paul Grossman, Basel; Dr. Gunver Kienle, Freiburg i.Br.; Dr. Mathias Bertram, Herdecke; Christine Widmer, St. Gallen.

Das Impulsreferat und der Workshop «Methodologie» ermöglichten die nötige wissenschaftliche Grundlage und Qualität, die das gesamte Symposium begleiteten. Interessanterweise wurde der umgekehrte Weg der Therapieentwicklung in der konventionellen Medizin (Screening von chemischen Substanzen $\rightarrow$ klinische Anwendung) mit dem der Komplementärmedizin (klinische Anwendung $\rightarrow$ biologische Mechanismen) verglichen. Insbesondere wurde die Frage nach angebrachten Komplementärmedizin- und Onkologie-gerechten Studiendesigns gestellt. Auch wurden beispielsweise praxisrelevante Designs diskutiert, wie die pragmatischen Studien ("pragmatic studies»), die die Evaluation von komplexen Therapien bei heterogenen Patientengruppen ermöglichen (Extension of the CONSORT Statement, 2008). 


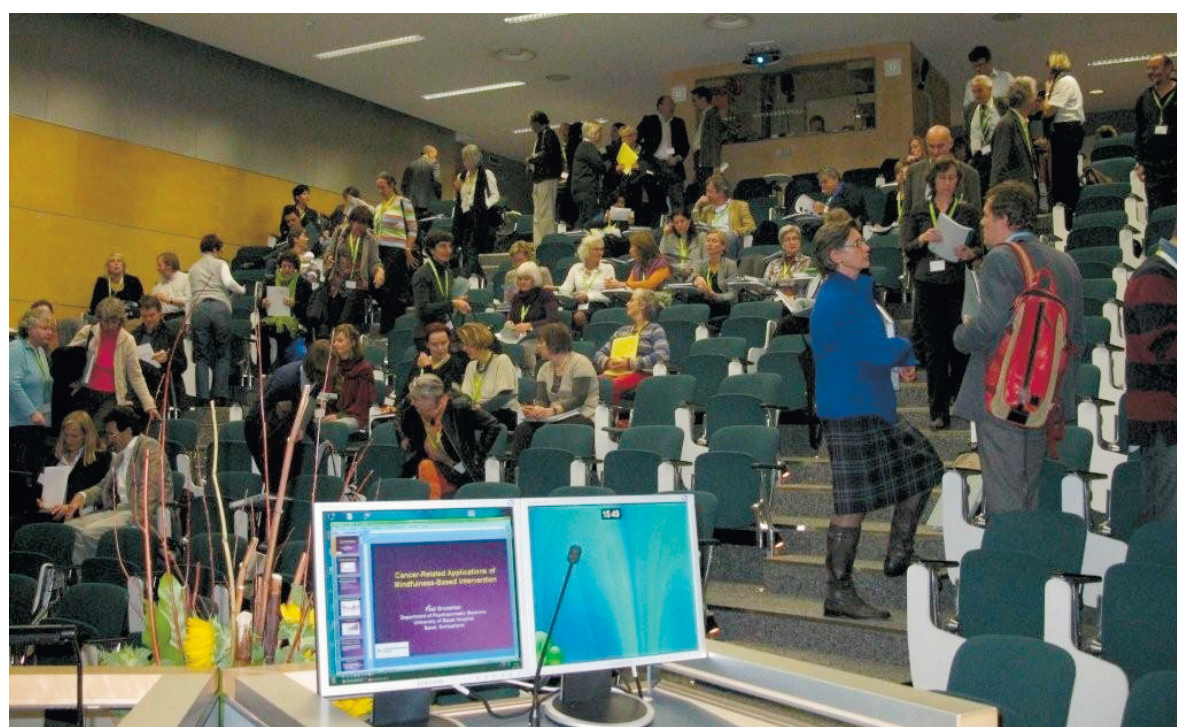

Der Workshop «Misteltherapie» identifizierte folgende Themen, die (verstärkt) beforscht werden sollten: Relevanz von Surrogatparametern für die Überlebenszeit, die Bedeutung der Lymphozyten als Steuerungsparameter, Untersuchungen in palliativer, rasch progredienter Situation, wie beispielsweise beim Pankreaskarzinom, Untersuchungen beim Mammakarzinom oder kolorektalen Karzinom in der adjuvanten Situation, die Kombination verschiedener Dosierungen und Applikationen sowie die Verlaufsbeobachtung und Dokumentation von Patienten, die keine konventionelle onkologische Therapie durchführen möchten.

Im Workshop «Kunsttherapien» wurden folgende Fragestellungen formuliert: Welche Formen der Künstlerischen Therapien sollten in der Onkologie verstärkt untersucht werden? In welchem onkologischen Setting haben sich Künstlerische Therapien besonders bewährt und können deswegen besonders gut ihre Wirksamkeit nachweisen? Wäre es sinn- voll, Therapiesysteme mit und ohne Künstlerische Therapien zu vergleichen? Welche Studiendesigns sind in der Onkologie effizient und ökonomisch machbar? Verbessert eine kunsttherapeutische Patientenschulung die Autonomie, Lebensqualität, Angst und Depressivität gegenüber alleiniger Standardtherapie? Gewünscht sind unter anderem randomisierte kontrollierte Studien.

Im Workshop «Pflege» wurde diskutiert, welche Voraussetzungen es brauche, damit Outcome-Messungen in der Integrativen Pflege möglich werden. Als ein mögliches Forschungsprojekt wurde eine Partizipation an einem Gesamtforschungsprojekt angesehen. Ein erster konkreter Schritt nach dem Symposium ist die Planung der Etablierung einer Internetplattform (E-Campus) für Forschung in der Integrativen Pflege.

Im Workshop «Akupunktur» wurde der Blick zunächst auf die Vorgehensweisen «systemorientiert versus symptomorientiert» in der Onkologie gelenkt und danach wurden klinisch relevante Indikationen ermittelt, wie zum Beispiel die Xerostomie, chemotherapieinduzierte Übelkeit oder Diarrhoe, Schmerz und Fatigue. Als ein Ziel wurde die Erarbeitung von Behandlungsrichtlinien im Konsens zwischen in der Akupunktur tätigem Fachpersonal und onkologischem Fachpersonal definiert.

Im Workshop «Achtsamkeit in der Onkologie» («mindfulness based intervention», MBI) wurde die Frage der Auswirkung einer MBI-Personalschulung (Ärzte, Pflege) gestellt hinsichtlich: Arbeitsplatzzufriedenheit, persönlichem Umgang mit Stress und nicht zuletzt Interaktion zwischen Personal und Patient.

Mit über 100 Teilnehmenden war das Symposium sehr gut besucht und zeigt, dass das Thema Integrative Onkologie und Forschung einem Bedürfnis der in der Onkologie tätigen Fachpersonen entspricht.

Das 2. Symposium wird vom 29.-30. November 2010 im Kantonsspital St. Gallen stattfinden. Weitere Informationen finden sich auf www.integrative-oncology.ch.

\section{Literatur}

1 Molassiotis A, Fernadez-Ortega P, Pud D, Ozden G, Scott JA, Panteli V, Margulies A, Browall M, Magri M, Selvekerova S, Madsen E, Milovics L, Bruyns I, Gudmundsdottir G, Hummerston S, Ahmad AM, Platin N, Kearney N, Patiraki E: Use of complementary and alternative medicine in cancer patients: a European survey. Ann Oncol 2005;16:655-663.

12 Wolf U, Maxion-Bergemann S, Bornhöft G, Matthiessen PF, Wolf M: Use of complementary medicine in Switzerland. Forsch Komplementmed 2006;13(suppl 2):4-6.

3 Leis AM, Weeks LC, Verhoef MJ: Principles to guide integrative oncology and the development of an evidence base. Curr Oncol 2008;15(suppl 2):s83-87. 